\title{
Pattern formation during mesophase growth in a homologous series
}

\author{
T. Tóth-Katona, T. Börzsönyi, Z. Váradi, J. Szabon, and Á. Buka \\ Research Institute for Solid State Physics of the Hungarian Academy of Sciences, H-1525 Budapest, P.O. Box 49, Hungary \\ R. González-Cinca and L. Ramírez-Piscina \\ Departament de Física Aplicada, Universitat Politècnica de Catalunya Campus Nord-Ed. B5, J. Girona Salgado s/n, \\ E-08034 Barcelona, Spain \\ J. Casademunt and A. Hernández-Machado \\ Departament d'Estructura i Constituents de la Matéria, Facultat de Física, Universitat de Barcelona, Diagonal 647, E-08028 Barcelona, \\ Spain \\ (Received 27 February 1996)
}

\begin{abstract}
Remarkable differences in the shape of the nematic-smectic- $B$ interface in a quasi-two-dimensional geometry have been experimentally observed in three liquid crystals of very similar molecular structure, i.e., neighboring members of a homologous series. In the thermal equilibrium of the two mesophases a faceted rectanglelike shape was observed with considerably different shape anisotropies for the three homologs. Various morphologies such as dendritic, dendriticlike, and faceted shapes of the rapidly growing smectic- $B$ germ were also observed for the three substances. Experimental results were compared with computer simulations based on the phase field model. The pattern forming behavior of a binary mixture of two homologs was also studied. [S1063-651X(96)04208-0]
\end{abstract}

PACS number(s): 61.30.-v, 64.70.Md, 68.10.Cr, 81.30.Fb

\section{INTRODUCTION}

The growth of crystals from their undercooled melt is one of the most interesting pattern forming phenomena. This nonequilibrium process can give rise to many different growing morphologies (e.g., dense branching, dendritic, faceted, or needle form) depending on the control parameter (undercooling) and on the magnitude and anisotropy of the material parameters (e.g., surface tension, kinetic, and diffusion coefficients).

The mathematical description of the crystallization that predicts the motion of the solidification front is rather complicated to handle both analytically and numerically. The basic ingredient is a scalar field, in this case the temperature $T(r, t)$, which satisfies the diffusion equation. The condition of heat conservation at a point on the moving interface determines the velocity of the front at that location for a given temperature field. This driving force arising from the effect of the temperature gradient near the solid-liquid boundary tends to destabilize the interface. The interfacial dynamics is taken into consideration by introducing the surface tension and the surface kinetics into the boundary condition. The competition between the stabilizing and destabilizing effects results in different growth morphologies for different sets of the relevant parameters.

Different models have been used in computer simulations, where by varying the value of the control parameter or that of the material parameters (and their anisotropy) one can construct the morphological phase diagram of the system. The boundary layer model simplifies the diffusion problem by assuming that the entire temperature change occurs within a narrow layer near the interface $[1,2]$. A detailed study of the behavior of the dendritic and dense branching morphologies was also made by using the diffusion-transition scheme $[3,4]$.
Some computer simulations employing phase field model [5-8] have been carried out recently. In these models an auxiliary field playing the role of a local order parameter locates the two different phases. This phase field (described in Sec. III) changes continuously across the interface, which therefore has finite width, contrary to the sharp-interface models.

A great number of experiments were done on traditional solid-liquid systems [9-13]. Different growth morphologies were observed in columnar liquid crystals [14-17] and also in low-molecular-weight liquid crystals [18-21]. Some of the growth morphologies on a nematic-smectic- $B$ interface reported in [21] were seen even in a quite different system of diluted ${ }^{3} \mathrm{He}-{ }^{4} \mathrm{He}$ solutions [22].

As reported earlier [21], the same sequence of morphologies of the growing smectic- $B$ germ was induced for onecomponent systems with very different molecular structure when changing the undercooling. In this paper we present results of experiments carried out on three substances of a homologous series, thus liquid crystals of similar molecular structure. One of them was studied extensively before $[21,23]$. The material parameters of the three homologs were experimentally determined and found to be similar (Sec. II), except the surface tension anisotropy (Sec. IV A), which changed considerably from one substance to another. No information is available about the magnitude and anisotropy of the kinetic coefficient, which also influences the pattern formation process. In spite of the similarities in molecular mass, latent heat, specific heat, and lattice parameters, the pattern forming behavior of the homologues investigated was quite different (Sec. IV B). Contrary to the more traditional experimental situation, where the transition between different morphologies is induced by changing the undercooling, here the surface tension anisotropy is tuned while changing the length of the alkyl chain of the homologs and a significant change 
TABLE I. Material parameters of the investigated substances.

\begin{tabular}{|c|c|c|c|c|}
\hline Parameter & & $\mathrm{CCH} 3$ & $\mathrm{CCH} 4$ & $\mathrm{CCH} 5$ \\
\hline$m$ & & 3 & 4 & 5 \\
\hline$T_{N S}\left({ }^{\circ} \mathrm{C}\right)$ & & $(56.3)$ & $(53.1)$ & $(51.2)$ \\
\hline$M(\mathrm{~g} / \mathrm{mol})$ & & 233 & 247 & 261 \\
\hline$L\left(10^{3} \mathrm{~J} / \mathrm{kg}\right)$ & & $22 \pm 1$ & $22 \pm 1$ & $17 \pm 1$ \\
\hline \multirow{4}{*}{$c_{p}(\mathrm{~J} / \mathrm{kgK})$} & in the nematic phase & & & \\
\hline & & $1610 \pm 60$ & $1900 \pm 80$ & $1510 \pm 60$ \\
\hline & in the smectic- $B$ phase & & & \\
\hline & & $1460 \pm 60$ & $1720 \pm 70$ & $1200 \pm 50$ \\
\hline \multirow[t]{3}{*}{ Sm- $B$ lattice parameters $(\AA)^{\mathrm{a}}$} & $l$ & 16.5 & & 18.5 \\
\hline & $b$ & 27.3 & & 31.2 \\
\hline & $a$ & 5.7 & & 5.7 \\
\hline
\end{tabular}

${ }^{\mathrm{a} B r o w n s e y ~ a n d ~ L e a d b e t t e r ~[24] . ~}$

in the growth morphologies during the nematic-smectic- $B$ phase transition has been observed. During these experiments the undercooling has been varied in the same narrow range for each substance. In Sec. IV C the experimental results are compared with the computer simulation based on the phase field model. Furthermore, in order to tune the average molecular mass from 233 to 261 we have prepared the binary mixture of $\mathrm{CCH} 3$ and $\mathrm{CCH} 5$ at different concentrations. The pattern forming behavior of this mixture is described in Sec. IV D.

\section{EXPERIMENTAL SETUP AND MATERIAL PARAMETERS}

Three liquid crystalline substances with chemical structures (see below) differing only in the length of the alkyl chain have been studied. All of them have a first-order nematic $(N)$-smectic- $B(\mathrm{Sm}-B)$ phase transition at temperature $T_{N S}$ (Table I). The $T_{N S}$ values given in the parentheses indicate a monotropic $N-\mathrm{Sm}-B$ phase transition. The molecular structure is as follows:

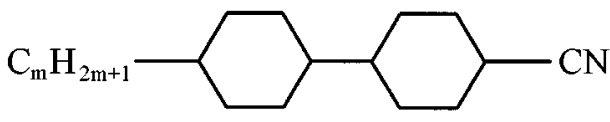

The relevant material parameters are given in Table I, where $m$ is the number of $\mathrm{C}$ atoms in the alkyl chain ( $m=3,4,5$ correspond to the labeling $\mathrm{CCH} 3, \mathrm{CCH} 4$, and $\mathrm{CCH} 5$, respectively), $M$ is the molecular weight, $L$ is the latent heat per unit mass of the $N-\mathrm{Sm}-B$ phase transition, $c_{p}$ is the specific heat per unit mass, $l$ is the molecular length, $b$ is the periodicity along the $\operatorname{Sm}-B$ director, and $a$ is the side length of the elementary cell in the hexagonal lattice of the $\mathrm{Sm}-B$ phase.

The X-ray-diffraction studies [24] showed that the Sm-B phase of $\mathrm{CCH} 3$ and $\mathrm{CCH} 5$ is of the same structure, with somewhat different lattice parameters. Both substances have bilayer structure associated with $A B A$... type layer packing without polar ordering. The interdigitation of the layers $l-b / 2 \approx 2.9 \AA$ for both substances, which is very close to the effective length of the $-\mathrm{CN}$ group (and that of the $-\mathrm{C}_{3} \mathrm{H}_{7}$ group). Note that the increase of the alkyl chain from $m=3$ to $m=5$ does not influence $a$.

$L$ and $c_{p}$ were measured by differential scanning calorimetry. For determination of $c_{p}$ an external sapphire standard was used, the specific heat of which is known as a function of temperature. The method included the scanning of the sapphire standard over the desired temperature range, making a blank determination, and afterward the scanning of the sample over the same range. The two curves were compared and $c_{p}$ was calculated. The $c_{p}$ values given in Table I correspond to temperatures close to $T_{N S}$, but still far enough, where the temperature dependence could be assumed to be linear.

The average heat diffusion coefficient $D$ and its anisotropy $D_{a}=\left(D_{\|}-D_{\perp}\right) / D_{\perp}\left(D_{\|}\right.$and $D_{\perp}$ are the diffusion coefficients parallel and perpendicular to the director, respectively) were not measured for the investigated substances. On the basis of the experimental determination of $D$ for some other liquid crystalline materials, we can assume some general features of the heat transport.

(i) $D_{a}>0$ for nematic liquid crystals and it remains positive also in smectic phases [25], contrary to the other transport anisotropies (e.g., mass transport and conductivity) for which the anisotropy changes sign in the smectic phases.

(ii) The magnitude of $D$ and that of $D_{a}$ in the Sm- $B$ phase do not differ significantly from those in the $N$ phase; see, for example [26,27].

(iii) Measurement on the alkylcyanobiphenyl liquid crystals [27] showed that the increase of the alkyl chain length by one or two carbon atoms does not affect the magnitude of $D$ significantly. On the other hand, increasing the chain length increases $D_{a}$. For example, increasing the aliphatic chains of azoxy benzene from $m=1$ ( $p$-azoxy anisol) to $m=8\left(4,4^{\prime}\right.$-di- $n$-octyloxy azoxy benzene) carbon atoms on both ends of the molecule, the $D_{a}$ increases from 0.5 to 1.7 [26]. Assuming a linear dependence of $D_{a}$ on $m$, this effect should decrease the heat diffusion anisotropy for $\mathrm{CCH} 3$ by about $11-12 \%$ compared to that of $\mathrm{CCH} 5$.

(iv) 4 ' $n$-pentyl-4-cyanobiphenyl, the molecular structure of which differs from $\mathrm{CCH} 5$ only in the rigid core (the cyclohexane rings of $\mathrm{CCH} 5$ are replaced by phenyl rings), 
has the experimentally determined values of the heat diffusion coefficients (in the nematic phase): $D_{\|}=(1.25 \pm 0.05) \times 10^{-3} \quad \mathrm{~cm}^{2} / \mathrm{s} \quad$ and $D_{\perp}=(0.79 \pm 0.04)$ $\times 10^{-3} \mathrm{~cm}^{2} / \mathrm{s}$ [26]. This leads to the heat diffusion anisotropy of $D_{a}=0.58$. Knowing that $D_{a}$ depends considerably only on the length of the rigid core [25], and assuming that replacement of the phenyl rings by cyclohexane does not lead to any drastic changes in the length, we could expect similar $D_{a}$ for CCH5.

In conclusion, for the investigated homologous series one would not expect any significant dependence of $D$ and $D_{a}$ on the temperature, phase (nematic or smectic), or substance (CCH3, CCH4, or CCH5). The only exception is possibly the anisotropy, which could be sensitive to $m$. However, its role on the pattern morphology is not yet clear.

The pattern formation during the $N-\mathrm{Sm}-B$ phase transition was observed in a quasi two-dimensional geometry. Planarly oriented liquid crystal cells of dimensions $1 \times 1 \mathrm{~cm}^{2}$ ( $x y$ plane) and of the thickness of $10 \mu \mathrm{m}$ ( $z$ direction) with polyamid coating and rubbing have been used. The nematic director $\mathbf{n}(N)$ was aligned along the $y$ direction. Twodimensional images (in $x y$ where the $N-\mathrm{Sm}-B$ interface appeared as a line) were observed by a polarizing microscope. The images were recorded onto videotape, digitized, and analyzed. The sample temperature was controlled in a hot stage with an accuracy of $0.002^{\circ} \mathrm{C}$. Other details of the experimental setup have been given elsewhere [28].

\section{PHASE FIELD MODEL FORMULATION}

In order to numerically simulate the experimental system, we have employed a thermodynamically consistent phase field model proposed by Wheeler et al. $[5,6,29,30]$ and applied to faceted growth in [8]. It is defined by two coupled nonlinear reaction-diffusion equations. Their dimensionless form is

$$
\begin{aligned}
& \epsilon^{2} \tau(\theta) \frac{\partial \phi}{\partial t}= \phi(1-\phi)\left[\phi-\frac{1}{2}+30 \epsilon \beta \Delta u \phi(1-\phi)\right] \\
&-\epsilon^{2} \frac{\partial}{\partial x}\left[\eta(\theta) \eta^{\prime}(\theta) \frac{\partial \phi}{\partial y}\right] \\
&+\epsilon^{2} \frac{\partial}{\partial y}\left[\eta(\theta) \eta^{\prime}(\theta) \frac{\partial \phi}{\partial x}\right] \\
&+\epsilon^{2} \nabla\left[\eta^{2}(\theta) \nabla \phi\right], \\
& \frac{\partial u}{\partial t}+\frac{1}{\Delta}\left(30 \phi^{2}-60 \phi^{3}+30 \phi^{4}\right) \frac{\partial \phi}{\partial t}=\nabla^{2} u,
\end{aligned}
$$

where $\phi$ is the phase field, changing across the interface from $\phi=0$ (smectic- $B$ ) to $\phi=1$ (nematic). Temperature is scaled by $T=T_{N S}+\Delta T u$, where $T_{N S}$ is the melting temperature and $\Delta T=T_{N S}-T_{\infty}$ is the undercooling, that is, the difference between $T_{N S}$ and the temperature at the boundaries of the system. Lengths are scaled in some arbitrary reference length $\omega$, while times are scaled by $\omega^{2} / D$, where $D$ is assumed the same in both phases. Directors of the nematic and smectic phases were taken parallel to each other.
The $x$ axis (which has been chosen perpendicular to the director) and the normal to the interface determine the angle $\theta$, while $\eta(\theta)=\sigma(\theta) / \sigma(0), \sigma(\theta)$ being the surface tension. Derivatives with respect to $\theta$ are denoted by primes. The dimensionless parameters of the model are

$$
\begin{gathered}
\Delta=\frac{c_{p} \Delta T}{L}, \\
\beta=\frac{\sqrt{2} \omega L^{2}}{12 c_{p} \sigma(0) T_{N S}}=\frac{\sqrt{2} \omega}{12 d_{o}}, \\
\epsilon=\frac{\delta}{\omega}, \\
\tau(\theta)=\frac{L D}{\sigma(0) T_{N S}} \frac{\eta(\theta)}{\mu(\theta)},
\end{gathered}
$$

where $c_{p}$ is the specific heat per unit volume, $L$ is the latent heat per unit volume, $d_{0}$ is the capillary length, $\delta$ is the interface thickness, and $\mu(\theta)$ is an anisotropic kinetic coefficient.

In phase field models the system is treated as a whole and no distinction is made between bulk and interface. In the limit of vanishing interfacial thickness, with $\epsilon \rightarrow 0$, the sharp interface model is recovered:

$$
\begin{gathered}
\frac{\partial T}{\partial t}=D \nabla^{2} T, \\
L v_{n}=D c_{p}\left[\left(\nabla_{n} T\right)_{\text {smectic }-B}-\left(\nabla_{n} T\right)_{\text {nematic }}\right], \\
T_{\text {interface }}=T_{N S}-\frac{T_{N S}}{L}\left[\sigma(\theta)+\sigma^{\prime \prime}(\theta)\right] \kappa-\frac{v_{n}}{\mu(\theta)},
\end{gathered}
$$

where $\nabla_{n}$ is the normal derivative at the interface, $v_{n}$ is the normal velocity of the interface, and $\kappa$ is the local curvature of the interface.

\section{RESULTS}

\section{A. Equilibrium shapes}

When undercooling the nematic phase, some smectic germs appear and grow rapidly until the whole substance gets into the Sm- $B$ phase. Then slowly heating up the sample and approaching $T_{N S}$, one can achieve a state when only a few Sm- $B$ islands surrounded by the $N$ phase are left and they are separated (usually far) from each other so that no interaction between them is present.

Choosing one of these smectic germs for further observation and controlling the temperature in order to keep the size of the germ constant, one can approach the thermal equilibrium state of this system. After typically $5-8 \mathrm{~h}$ of equilibration the shape of the $N-\mathrm{Sm}-B$ interface is stabilized and for each substance of the homologous series studied $(\mathrm{CCH} 3$, $\mathrm{CCH} 4$, and $\mathrm{CCH} 5$ ) it shows a faceted, rectanglelike, elongated shape [Figs. 1(a)-1(c)]. The longer, faceted edges are parallel to the smectic layers (perpendicular to the director). According to the minimum of the elastic free energy at the surface, the orientation of the $N$ and $\mathrm{Sm}-B$ directors $[\mathbf{n}(N)$ 

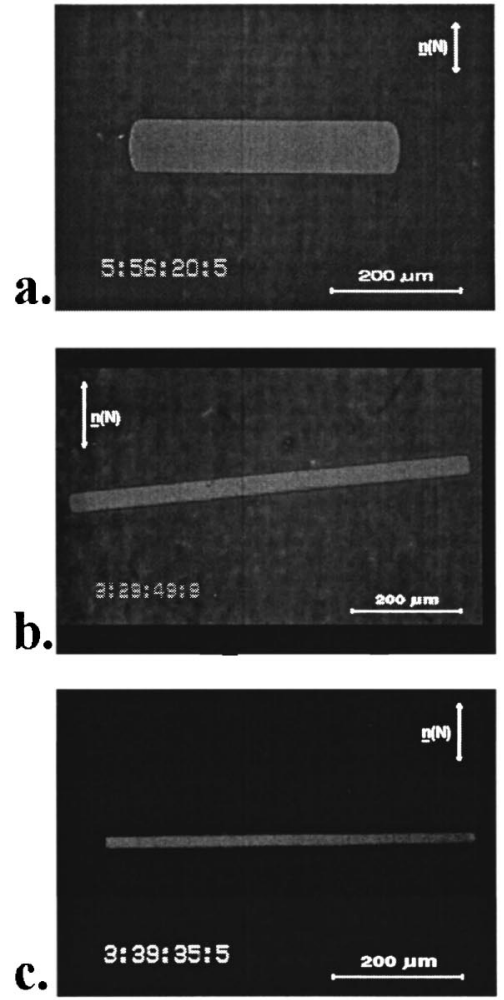
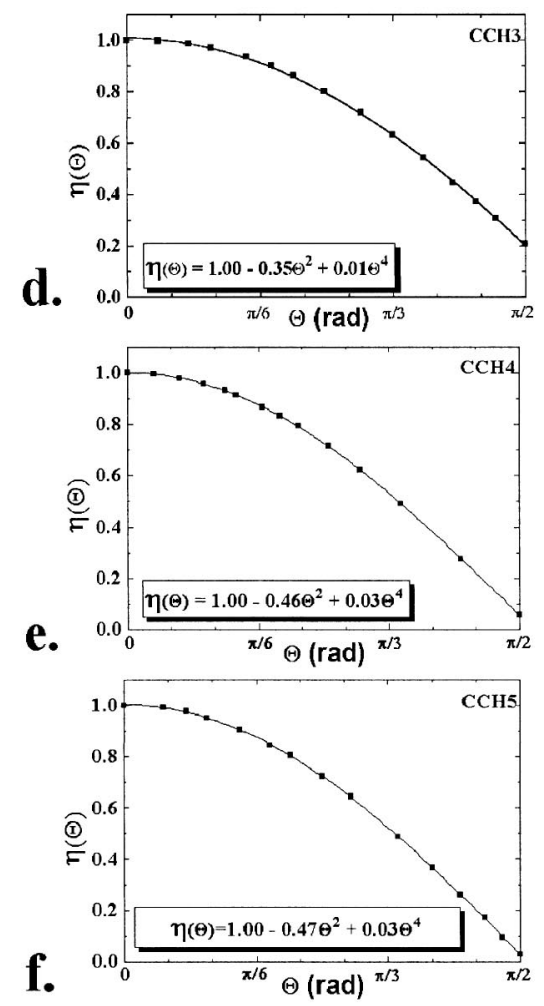

FIG. 1. Equilibrium shapes of the smectic- $B$ germ surrounded by the nematic for (a) $\mathrm{CCH} 3$, (b) $\mathrm{CCH} 4$, and (c) $\mathrm{CCH} 5$, and the angle dependence of the surface tension for (d) $\mathrm{CCH} 3$, (e) $\mathrm{CCH} 4$, and (f) $\mathrm{CCH} 5$ as a result of the Wulff construction based on (a) -(c) (dots). The solid lines represent a polinomial fit, the parameters of which are given in the plots.

and $\mathbf{n}(S)$, respectively] are parallel (no distortion of the director at the interface). This was observable in most experimental cases when a small Sm- $B$ germ was carefully equilibrated and grown adiabatically slowly up to its final size shown in Figs. 1(a) and 1(c). Deviations up to $10^{\circ}$ in the plane can occur [see Fig. 1(b)], if the slow growth was started with a large germ oriented initially tilted with respect to the $\mathbf{n}(N)$. Moreover, in the case of $\mathrm{CCH} 5$, besides the in-plane tilting, sometimes an out-of-plane tilt of the $\mathbf{n}(S)$ was also observed even up to an angle of $90^{\circ}$ between $\mathbf{n}(N)$ and $\mathbf{n}(S)$, which corresponds to a homeotropically aligned smectic liquid crystal surrounded with the planar nematic liquid crystal. The shape of the homeotropic germ was found to be nonfaceted; it has a nearly circular shape that shows a slight hexagonal modulation [21]. Out-of-plane tilted (tilt angle less than $90^{\circ}$ ) germs are oval. In this paper we focus our attention only on the planar smectic germs.

The shape anisotropy of the equilibrium germ is unexpectedly different for the three homologs of the series. The length to width ratio $\eta^{-1}(\pi / 2)$ of the germs differs about an order of magnitude and is $\sim 4.8$ for $\mathrm{CCH} 3, \sim 18$ for $\mathrm{CCH} 4$, and $\sim 36$ for $\mathrm{CCH} 5$ (within a scattering of $20 \%$ from germ to germ).

The Wulff construction [31,32] enables one to determine the angle dependence of the surface tension of the system by evaluating the equilibrium shape of the interface. This is a geometrical construction based on the idea that in thermal equilibrium the surface free energy is minimized. The Wulff plots [the angular dependence of the surface tension $\eta(\theta)$ ], obtained from the equilibrium shapes, can be seen in
Figs. 1(d) $-1(\mathrm{f}) . \theta=0$ corresponds to the direction of the longer edges.

If one extends the range of $\theta$ to $2 \pi$ one gets cusps at $\theta=\pi / 2,3 \pi / 2$ in the curve of the surface tension function, which correspond to faceting of the longer edges. The solid lines in Figs. 1(d)-1(f) are fitted curves with parameters shown in the plots. A small deviation in the coefficient of $\theta^{4}$ in Fig. 1(d) from the earlier published data [21] occurs because of neglecting the higher-order terms here. The surface tension anisotropy $[1-\eta(\pi / 2)] /[1+\eta(\pi / 2)]$, which actually corresponds to the shape anisotropy of the germ, is the smallest $(0.68)$ for $\mathrm{CCH} 3$ and gets larger $(0.89)$ for $\mathrm{CCH} 4$ and (0.94) for CCH5.

\section{B. Spontaneous nucleation}

Applying a large enough undercooling $\left(\Delta T \geqslant 0.3^{\circ} \mathrm{C}\right)$ to the nematic liquid crystal, spontaneously nucleated Sm- $B$ germs appear and grow very rapidly. Their shape is usually very different from the equilibrium one and is material specific for the homologous series studied (see Fig. 2), contrary to previously published cases [21] when substances with different molecular structure (two- or three-ring systems, biphenyl or bicyclohexane derivatives containing either cyano or trifluoromethoxyphenyl substituents) showed similar morphologies.

The orientation of the Sm- $B$ director is usually planar, thus it lies in the plane of the picture $\mathrm{CCH} 5$ is again the exception, showing in some cases homeotropic, or out-ofplane, tilted germs). In this section we will report the char- 

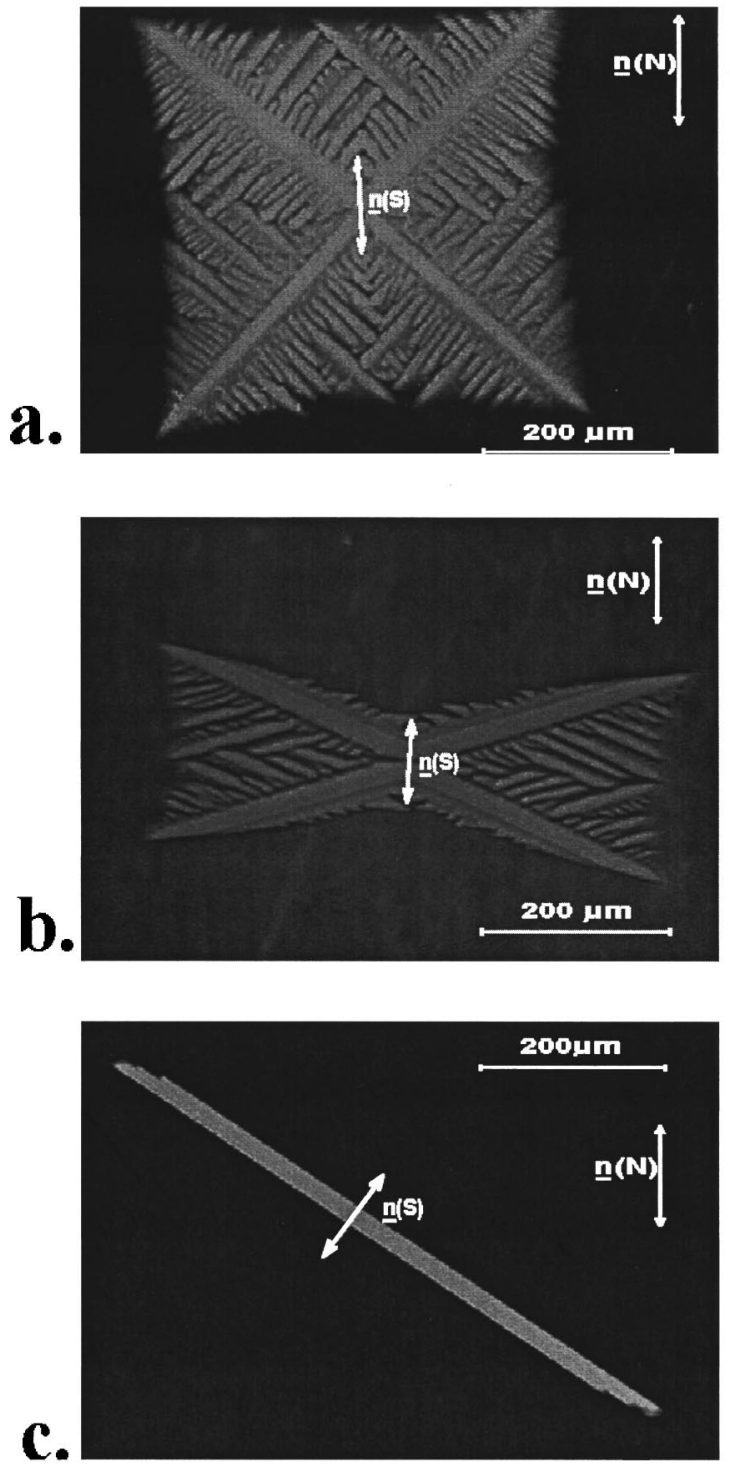

FIG. 2. Spontaneously nucleated smectic- $B$ germs for (a) $\mathrm{CCH} 3$, (b) $\mathrm{CCH} 4$, and (c) $\mathrm{CCH} 5\left(\Delta T=1.0^{\circ} \mathrm{C}\right) . \mathbf{n}(N)$ and $\mathbf{n}(S)$ indicate the nematic and the smectic director, respectively.

acteristics of the growing $\mathrm{Sm}-B$ germs, obtained by applying the undercooling $\Delta T=1.0^{\circ} \mathrm{C}$, which gives dimensionless values of $\Delta=0.073 \pm 0.004, \quad 0.086 \pm 0.005, \quad$ and $0.089 \pm 0.006$ for $\mathrm{CCH} 3, \mathrm{CCH} 4$, and $\mathrm{CCH} 5$, respectively. Experiments showed for each substance that the change of $\Delta$ in the range of $0.07-0.11$ does not influence the growth morphology of the interface for the given compound. The increasing undercooling in that range causes only a slight increase of the growth velocity $(v)$.

For quantitative characterization of the growing smectic structure the following labels were used (see also Fig. 3): $\alpha$ is the angle between the two closer main branches of the dendrite, which coincides with that of a main branch and its side branches, and $\gamma$ is the angle between the directors $\mathbf{n}(N)$ and $\mathbf{n}(S)$.

In the case of $\mathrm{CCH} 3$ we get dendritic growth with fourfold symmetry and parabolic tips. The angle between the main branches is $\alpha \approx 90^{\circ}$ and it is the same for each nucleation centre. A deviation less than $2^{\circ}$ from $\alpha=90^{\circ}$ was

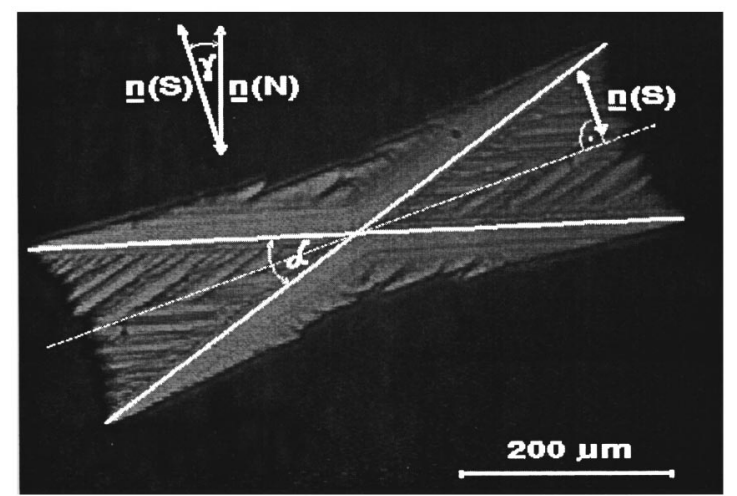

FIG. 3. Notations used for characterization of the smectic- $B$ growth morphology.

found for a large number of germs studied [see Fig. 4(d)]. Strong and symmetric sidebranching activity, even of second and third generation, is observed [see Fig. 2(a)]. The director of the nucleated Sm- $B$ germ is parallel to the director of the surrounding nematic liquid crystal $(\gamma \approx 0)$. In $93 \%$ of the 270 germs considered, $\gamma$ was found below $5^{\circ}$ and in the remaining $7 \%$ of them it was between $5^{\circ}$ and $9^{\circ}$ [see Fig. 4(a)].

The behavior of $\mathrm{CCH} 4$ is very unusual. We get dendriticlike growth, which means that the four main branches are still observable with intensive sidebranching, but the tips and the length of the side branches are not symmetric with respect to the direction of the normal growth velocity of the tip [see Fig. 2(b)]. The angle between the main branches is in the range $0^{\circ} \leqslant \alpha \leqslant 60^{\circ}$ and varies from germ to germ [see the distribution on Fig. 4(e)], but it does not seem to depend on the undercooling. As the number of measurements is relatively small, it is not possible to carry out a statistical analysis of the $\alpha$ distribution on Fig. 4(e). Still, Fig. 5 demonstrates the variety of the shapes that we obtain for $\mathrm{CCH} 4$.

The angle between the nematic and smectic directors $(\gamma)$ covers also a wide range: $0^{\circ} \leqslant \gamma \leqslant 60^{\circ}$. The distribution of $\gamma$ obtained for 270 germs is shown in Fig. 4(b). A large part $(\sim 37 \%)$ of the germs is still oriented parallel to $\mathbf{n}(N), \gamma=0$, but the rest are "disaligned" with respect to the nematic director, thus $\gamma$ reaches values up to $60^{\circ}$. No germs were found with extremely large disalignment in the range $60^{\circ}<\gamma<90^{\circ}$.

No correlation between $\alpha$, $\gamma$, and $v$ was found; moreover, $\alpha$ and $\gamma$ did not show any dependence on the undercooling $\Delta T$. In general, the measured quantities ( $\alpha, \gamma$, and $v$ ) of the growing germ depend strongly on the type of the nucleation point (impurities, orientational defects of the director, or defects on the bounding glass plates). When repeating the experiment several times taking care of not crystallizing the sample, the properties of the germ $(\alpha, \gamma, v)$ are reproducible for the same nucleation point. But when getting into the crystalline phase the characteristics of the nucleation point might also change, and after heating up the sample again, by carrying out the next experiment one might get different conditions at the same location as before.

Another interesting phenomenon was detected during the pattern growth in $\mathrm{CCH} 4$. The growing $\mathrm{Sm}-B$ germ has nonreflection symmetry for $\gamma \neq 0$. The pair of main branches 

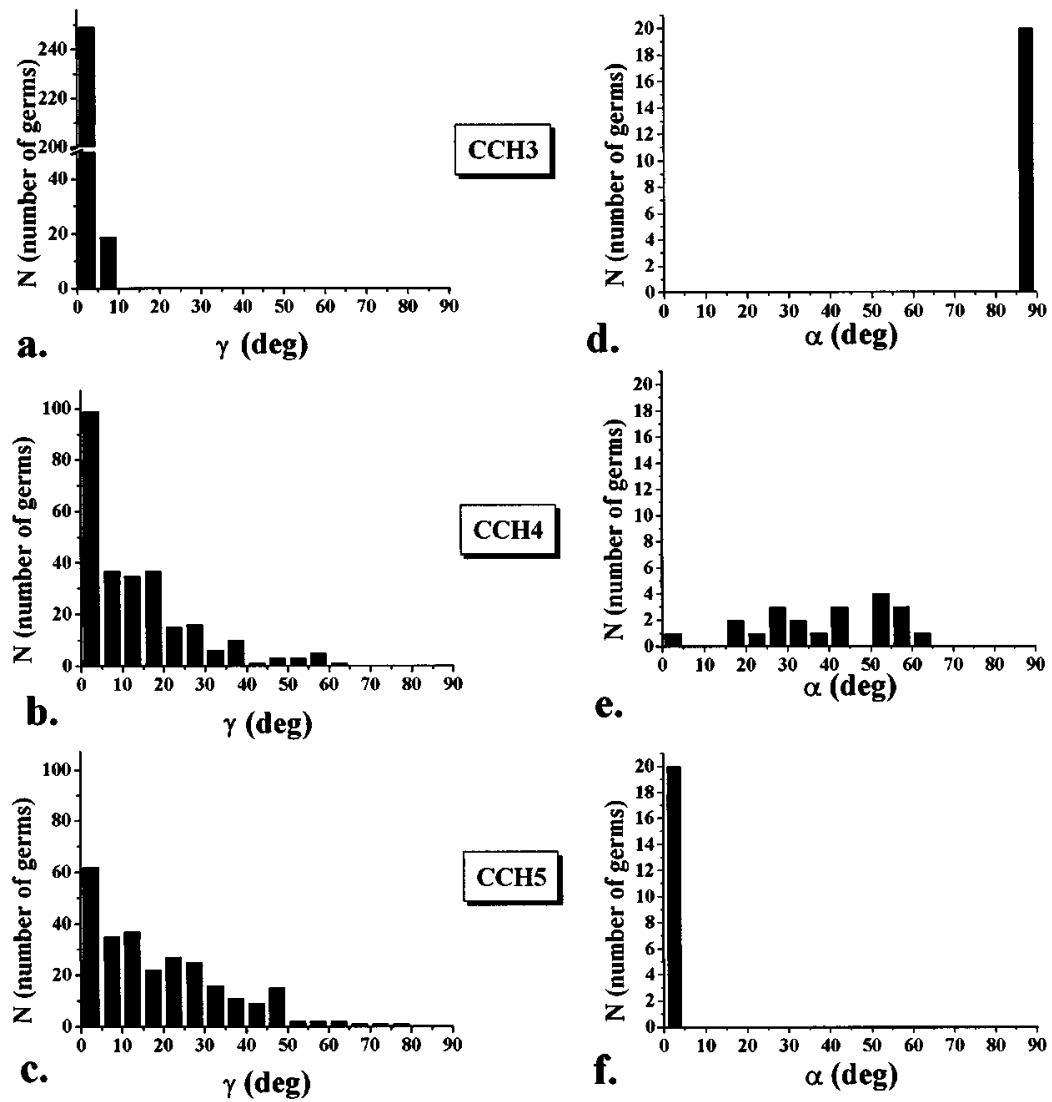

FIG. 4. Number of germs growing with a certain $\gamma$ for (a) $\mathrm{CCH} 3$, (b) $\mathrm{CCH} 4$, and (c) $\mathrm{CCH} 5$ and number of germs growing with a certain $\alpha$ for (d) $\mathrm{CCH} 3$, (e) $\mathrm{CCH} 4$, and (f) CCH5.

with opposite growth direction, which enclose larger angle with $\mathbf{n}(N)$, had larger growth velocity $v_{1}$ than the other pair $\left(v_{2}\right)$ (see Fig. 5). The experimentally observed relative difference in the growth velocities was up to $\delta v$ $=\left(v_{1}-v_{2}\right) / v_{1}=0.2$. In case of $\gamma=0$, i.e., when the four main branches make the same angle with $\mathbf{n}(N)$, the germ had symmetrical shape $(\delta v=0)$ for any value of $\alpha$.

For $\mathrm{CCH} 5$ the growing $\mathrm{Sm}-B$ germ has a faceted shape at any undercooling reminding its equilibrium shape, differing only at the short sides, which become unstable [compare
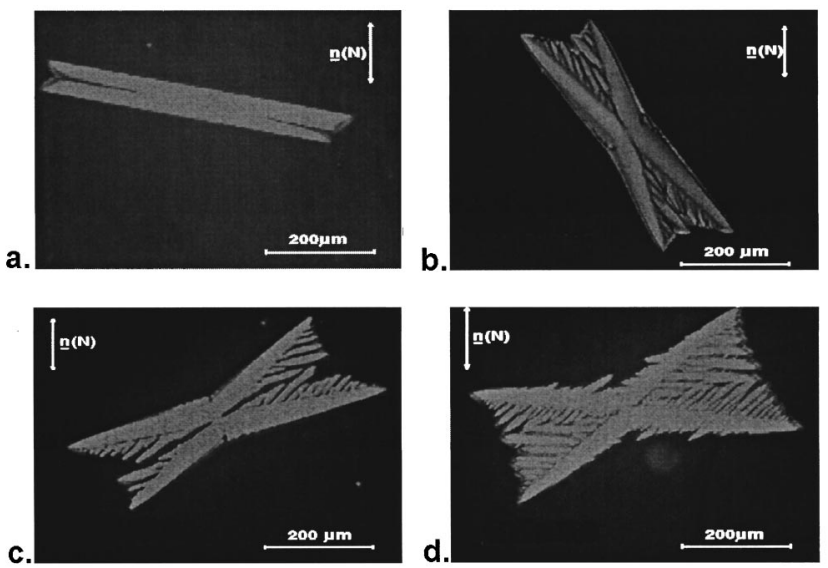

FIG. 5. (a)-(d) Different spontaneously nucleated smectic- $B$ germs for the substance $\mathrm{CCH} 4\left(\Delta T=1.0^{\circ} \mathrm{C}\right)$.
Figs. 1(c) and 2(c)]. This can be considered as a limit $\alpha=0^{\circ}$; see Fig. 4(f).

The directors of the $N$ and $\mathrm{Sm}-B$ phases show even weaker correlation than for $\mathrm{CCH} 4$. Here the distribution is broader $\left(0^{\circ} \leqslant \gamma \leqslant 80^{\circ}\right)$, but still germs with smaller $\gamma$ nucleate more often [Fig. 4(c)]. The $\gamma$ and $v$ of the nucleated Sm$B$ germ at the same location in the sample might change after crystallization in the same way as it was described above for CCH4.

\section{Comparison of the experiments and simulation}

The morphologies corresponding to the growth of germs of $\mathrm{CCH} 3, \mathrm{CCH} 4$, and $\mathrm{CCH} 5$ have been reproduced by simulation of the phase field model. In order to do that, a discretized version of the phase field equations (1) and (2) was numerically integrated in a uniform square lattice of $300 \times 300$ grid points with mesh spacing $\Delta x$ by using firstorder finite differences. The explicit time-differencing scheme applied to the $\phi$ equation forced us to adjust the value of $\Delta t$ in order to avoid numerical instability. The heat equation was solved using the unconditionally stable alternating-direction implicit method. Simulations reproducing the morphologies corresponding to substances $\mathrm{CCH} 3$ and $\mathrm{CCH} 4$ have been presented elsewhere [8] and showed remarkably good agreement with experimental morphologies in the different regimes obtained for different undercoolings. 


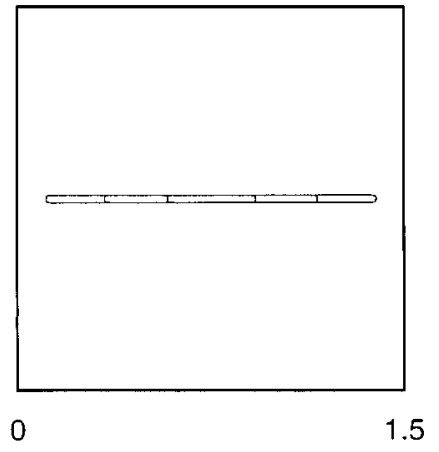

FIG. 6. Phase field model simulation of the nematic-smectic$B$ interface in $\mathrm{CCH} 5$, using $\Delta=0.11,300 \times 300$ grid points, $\Delta x=\epsilon=0.005, \beta=350, n=20$, and $\Delta t=9 \times 10^{-6}$. The times corresponding to the different contours of the interface are $t=0.036,0.090,0.144$

The slow growth regime of CCH5 (Fig. 6) has been reproduced locating the smectic- $B$ seed in the center of the computational system. Values of $\phi$ and $u$ were initially set equal to 1 and -1 , respectively, in the whole system except in its center, where $\phi=0$ and $u=0$. The kinetic term was taken to be isotropic, which implies a relation $\tau(\theta)=n \eta(\theta)$ with constant $n$. The anisotropy of the surface tension shown in Fig. 1(f) has been used. The parameters $\beta, \epsilon$, and $n$ were adjusted after some test runs. By choosing $\omega$ as large as the longest relevant spatial scale (determined by the thermal diffusion), $\beta$ has to be taken very large. The dimensionless parameter $\epsilon$ must be sufficiently small in order to reproduce the structure and to approximate the sharp interface limit. A satisfactory numerical accuracy is obtained when $\Delta x$ and $\epsilon$ have similar values. We have used $\Delta x=0.005, \epsilon=0.005, \beta=350, n=20, \Delta t=9 \times 10^{-6}$, and $\Delta=0.11$. A rectanglelike shape is observed with a faceted side much larger than the rough one. The distance between both faceted sides remain constant during all the simulation time. The shape is very similar to what was observed in the equilibrium experiments; see Fig. 1(c).

The CCH5 morphology corresponding to spontaneous nucleation (Fig. 7) has been computationally reproduced using the same parameters as in the previous case except $\Delta$, the new value of which is 0.16 , and $\Delta t=8 \times 10^{-6}$. The computational system used corresponds to one-quarter of the whole experimental system, being the germ set in the lower left corner. The same anisotropy of the surface tension used in the slow growth regime simulations and also an isotropic kinetic coefficient have been considered. A small perturba-

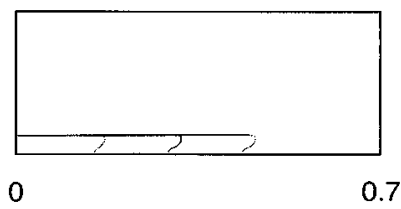

FIG. 7. Phase field model simulation of the spontaneous nucleation in $\mathrm{CCH} 5$. One-quarter of the nematic-smectic- $B$ interface is represented using $\Delta=0.16,300 \times 300$ grid points, $\Delta x=\epsilon=0.005$, $\beta=350, n=20$, and $\Delta t=8 \times 10^{-6}$. The times corresponding to the different contours of the interface are $t=0.035,0.070,0.105$. tion on the rough side is observable in Fig. 7. The distance between the faceted sides is now larger than in the slow growth regime, in accordance with the experiments [compare Figs. 7 and 2(c)]. However, it is observed here that the short ends split up, instead of a steplike form, seen experimentally.

\section{Properties of the mixture of $\mathrm{CCH} 3$ and $\mathrm{CCH5}$}

As described above, an unexpected large difference (about one order of magnitude) was detected between the surface tension anisotropies (gained from the equilibrium shape) of the two single compounds $\mathrm{CCH} 3$ and $\mathrm{CCH} 5$. As a consequence, their growth morphology and dynamics is also remarkably different. The substance $\mathrm{CCH} 4$ showed an unusual behavior, which could be interpreted as being intermediate between $\mathrm{CCH} 3$ and $\mathrm{CCH} 5$. In order to get a better understanding, a "virtual CCH4" was constructed by mixing $\mathrm{CCH} 3$ and $\mathrm{CCH} 5$ and the intermediate homolog was experimentally "simulated" by varying the concentration from pure $\mathrm{CCH} 3$ to pure $\mathrm{CCH} 5$.

Mixtures of $10,20, \ldots, 90$ mole $\%$ have been prepared. In order to ensure similar conditions, the same planarly oriented cells of $10 \mu \mathrm{m}$ thickness have been used as for the onecomponent substances.

The binary mixture showed a similar phase sequence on cooling $(I-N-\mathrm{Sm}-B)$ in the whole concentration range as its components. The $c-T$ (concentration-temperature) diagram of the system obeys continuous mixing at high temperatures resulting in a linear $T_{N I}(c)$. On the other hand, a negative deviation (up to $10^{\circ} \mathrm{C}$ ) of $T_{N S}(c)$ from the additivity rule could be detected; see Fig. 8(a). The filled squares on the figure denote the temperatures where the $\mathrm{Sm}-B$ phase was completely melted, thus the liquidus curve. The figure also indicates $T_{N S}$ for the pure $\mathrm{CCH} 4$.

The pattern formation of the spontaneously nucleated Sm$B$ phase in the nematic surrounding was studied for different concentrations and undercoolings in planar orientation of the both phases. The pattern forming behavior of the mixture showed similarities to that of the $\mathrm{CCH} 4$, which supports the idea that the mixture can account for the virtual singlecomponent $\mathrm{CCH} 4$.

In the concentration range $0-10 \%$ of $\mathrm{CCH} 5$ the growth morphologies were similar to the characteristic ones of $\mathrm{CCH} 3$. The angle between the four main branches was about $90^{\circ}$, with the largest deviation of $10^{\circ}$. The strong correlation between the $N$ and Sm- $B$ directors was found in these samples, i.e., $\gamma \approx 0$, similarly to $\mathrm{CCH} 3$.

In a wide concentration range (10-60\% of CCH5) we observed dendriticlike growth, where the angle between the main branches $\alpha$ varied from $90^{\circ}$ to $0^{\circ}$ [see Fig. 8(b)]. Different values of $\alpha$ in Fig. 8(b) for a given concentration were obtained for different germs and are not originated from experimental errors. Similarly to the case of $\mathrm{CCH} 4$, the values of $\alpha$ and $\gamma$ depend strongly on the nucleation point. The only difference is that in the mixture $\alpha$ showed a slight dependence on the undercooling. For larger $\Delta T$, smaller $\alpha$ was observed [see Fig. 8(b)]. In the range $60-100 \%$ of CCH5 the morphology was similar to that of the $\mathrm{CCH} 5$, i.e., $\alpha=0$ and $\gamma$ varied in a wide range from germ to germ. 

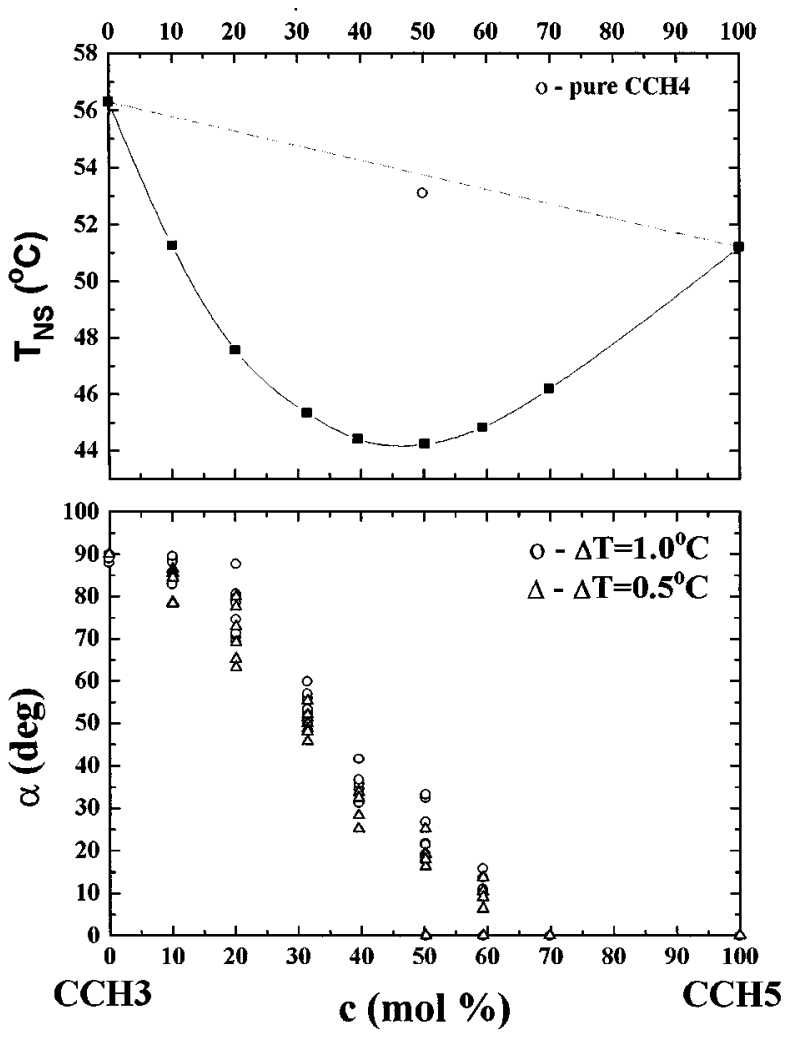

FIG. 8. (a) Concentration-temperature diagram for the binary system $\mathrm{CCH} 3-\mathrm{CCH} 5$ and (b) concentration dependence of the angle $\alpha$.

Concerning growth velocities $v$, generally much smaller values have been found in the mixtures than in onecomponent materials. For $\Delta T=1.0^{\circ} \mathrm{C}$ the average growth velocities of 515,174 , and $177 \mu \mathrm{m} / \mathrm{s}$ have been measured for $\mathrm{CCH} 3, \mathrm{CCH} 4$, and $\mathrm{CCH} 5$, respectively [33]. Adding $\mathrm{CCH} 5$ to $\mathrm{CCH} 3$ even in small concentrations $(\sim 10$ mole $\%$ of CCH5) decreased the $v$ drastically (by about an order of magnitude) compared to the one-component $\mathrm{CCH} 3$. The further increase of the $\mathrm{CCH} 5$ concentration in the mixture from $10 \%$ to $70 \%$ did not seem to influence the $v$ significantly. For example, in 50\% CCH3 - 50\% CCH5 mixture, the average velocity was found to be about $47 \mu \mathrm{m} / \mathrm{s}$, which is not very different from that measured in $90 \% \mathrm{CCH} 3-10 \%$ CCH5 system $(38 \mu \mathrm{m} / \mathrm{s})$, but it is much lower than that determined for the one-component $\mathrm{CCH} 3, \mathrm{CCH} 4$, and CCH5.

\section{CONCLUDING REMARKS}

The equilibrium shape of the interface between the nematic-smectic- $B$ phases was found to be faceted and rectanglelike for each member of the homologous series, but with very different length to width ratios (see Fig. 1). The surface tension anisotropy is one material parameter that shows a relevant variation while changing the length of the alkyl chain. The significant difference between $\mathrm{CCH} 3$ and CCH5 in the surface tension anisotropy could be explained by the molecular packing of the $\mathrm{Sm}-B$ phase. In the case of $\mathrm{CCH} 3$ there is no difference in the length of the two end groups of the molecule $\left(\mathrm{CN}\right.$ and $\mathrm{C}_{3} \mathrm{H}_{7} \approx 3 \AA$ ), while for CCH5 the alkyl chain is longer $\left(\mathrm{C}_{5} \mathrm{H}_{11} \approx 5 \AA\right)$. Since the molecules form layers in the smectic- $B$ phase and have between attaching neighboring layers random end-to-end packing [24], the faceted interface (parallel to the smectic layers) of the equilibrated smectic- $B$ domain is rougher on molecular scale for $\mathrm{CCH} 5$ than for $\mathrm{CCH} 3$. Because of this roughness the packing between the $N$ and $\mathrm{Sm}-B$ phases is presumably better for $\mathrm{CCH} 5$, which leads to a smaller surface tension on the faceted sides. For the substance $\mathrm{CCH} 4$ one expects an intermediate value, which is observed experimentally.

A drastic change in the growing morphology of the spontaneously nucleated smectic- $B$ germs has also been detected by varying the length of the alkyl chain (see Fig. 2). While in $\mathrm{CCH} 3$ a dendritic shape with fourfold symmetry with $\alpha \approx 90^{\circ}$ was observed, the substance CCH5 showed only a small deviation from its equilibrium shape, thus an elongated rectangle. In the case of $\mathrm{CCH} 4$, an intermediate dendriticlike shape appeared with $0^{\circ} \leqslant \alpha \leqslant 60^{\circ}$. One explanation could be a large difference in $D$. A larger $D$ for CCH5 compared to the $\mathrm{CCH} 3$, leaving the other material parameters similar (which is a realistic assumption; see Table I), would stabilize the interface. However, on the basis of the analysis described in Sec. II, we would not expect any significant difference in $D$ for $\mathrm{CCH} 3$ and $\mathrm{CCH} 5$. On the other hand, phase field model simulations, using the same $D$ and the experimentally determined surface tension anisotropies (neglecting the anisotropy of the kinetic coefficient and that of the diffusion coefficient), reproduced qualitatively the growth morphologies for CCH4 [8] and for CCH5 observed experimentally. For CCH5 the surface tension anisotropy is large (and the cusp in the angular dependence of the surface tension is deep), which presumably does not allow the destabilization of the faceted sides. Only the short rough sides are destabilized, as seen in experiments [Fig. 2(c)] and also in simulations (Fig. 7). Computer simulations involving only the surface tension anisotropy did not reproduce completely the qualitative picture of the experimental situation for $\mathrm{CCH} 3$, which has the lowest surface tension anisotropy among all investigated substances. First of all, the experimentally determined $\alpha=90^{\circ}$ could not be reached. In order to have a better understanding of the experimentally determined growth morphology, we should probably take into account the anisotropy of the kinetic coefficient and that of the diffusion coefficient. Computer simulations [8] showed that the angle $\alpha$ is very sensitive to the angular dependence of the kinetic term.

The asymmetry of the sidebranching activity with respect to the growth direction of the main tip in case of $\mathrm{CCH} 4$ [see, for example, Figs. 2(b) and 3], which has been observed both experimentally and by computer simulation, could be understood by taking into account the angle between the smectic$B$ director and the normal to the interface. In the vicinity of the tip on that part of the interface where its normal encloses a smaller angle with the smectic director (external side; see Fig. 3) the interface is more stable against the perturbations than on the other (internal) side. This is due to the fact that the coincidence of the surface normal and the director corresponds to the smallest surface tension, which means that for this part of the interface it is not easy to roughen up, compared to other parts where the angle between the surface 
normal and the director is larger. It is also clearly visible on Figs. 2(b), 3, and 5 that on the external side of the dendritic tip the secondary arms easily became faceted, with facets parallel to the smectic layers. This causes a time-dependent growth direction of the secondary-arm tip. These phenomena are in accordance with the observation that the tendency during the crystallization is the expansion of the already existing smectic layers instead of the creation of new ones.

The different growth velocities of the main branches (nonreflection symmetry of the dendrites) observed in the case of $\mathrm{CCH} 4$ could be possibly also explained by taking into consideration the anisotropy of the heat diffusion coefficient and that of the kinetic term. Some preliminary simulations with an anisotropic heat diffusion showed such a nonreflection symmetry of the dendrites.

By the experimental studies of the binary mixture $\mathrm{CCH} 3-$ $\mathrm{CCH} 5$ in various concentrations, the "intermediate" behavior of CCH4 was succesfully "simulated" regarding the angle $\alpha$ between the main branches. A continuous decrease of $\alpha$ was found by increasing the CCH5 concentration. On the other hand, the mixture did not behave like $\mathrm{CCH} 4$ concerning the growth velocity and the sidebranching. The observed weaker sidebranching activity (large wavelength, slow growth without second generation of the side branches) in the mixtures compared to that of $\mathrm{CCH} 4$ was the consequence of the decreased $v$. Observations such as negative deviation of $T_{N S}(c)$ from the additivity rule, large temperature range of the $N$ and $\mathrm{Sm}-B$ phase coexistence (up to $\approx 2^{\circ} \mathrm{C}$ ), drastic decrease in the main branch growth velocity (by about one order of magnitude) compared to the one- component substances, and the increased nucleation rate warn us that the mixing of the two compounds is presumably not ideal. All these facts lead us to conclude that for a detailed quantitative analysis, besides the heat diffusion, we should also consider the mass transport. This leads to further complications because in liquid crystals, the mass diffusion coefficient is not only several orders of magnitude smaller than that of heat diffusion, but the mass diffusion anisotropy changes sign at the $N-\mathrm{Sm}-B$ transition [34], contrary to the heat diffusivity anisotropy.

\section{ACKNOWLEDGMENTS}

The authors wish to thank Professor L. Kramer for many fruitful discussions and A. Vajda for preparation of the mixtures. R.G.C. is indebted to the Research Institute for Solid State Physics, Budapest for its kind hospitality and assistance. A.B. appreciates the support and kind hospitality of the University of Barcelona and Politechnical University of Catalunya. This cooperation stimulated the EU network proposal No. ERB 4061 PL 95-1377. Investigated substances were kindly made available for us by Merck, Darmstadt. The work was financially supported by the Hungarian Academy of Sciences Grant No. OTKA T014957, the Volkswagen Foundation, and the Dirección General de Investigación Científica y Técnica, Projects Nos. PB93-0769-C02-02 and PB93-0054-C02-01. We also acknowledge the Centre de Supercomputació de Catalunya for computing support.
[1] E. Ben-Jacob and P. Garik, Physica D 38, 16 (1989).

[2] E. Ben-Jacob and P. Garik, Nature 343, 523 (1990).

[3] O. Shochet, K. Kassner, E. Ben-Jacob, S. Lipson, and H. Müller-Krumbhaar, Physica A 181, 136 (1992).

[4] O. Shochet, K. Kassner, E. Ben-Jacob, S. Lipson, and H. Müller-Krumbhaar, Physica A 187, 87 (1992).

[5] G. McFadden, A. Wheeler, R. Braun, S. Coriell, and R. Sekerka, Phys. Rev. E 48, 2016 (1993).

[6] A. Wheeler, B. Murray, and R. Schaefer, Physica D 66, 243 (1993).

[7] R. Kobayashi, Physica D 63, 410 (1993).

[8] R. González-Cinca, L. Ramírez-Piscina, J. Casademunt, A. Hernández-Machado, L. Kramer, T. Tóth Katona, T. Börzsönyi, and A. Buka, Physica D (to be published).

[9] K. Koo, R. Ananth, and W. Gill, Phys. Rev. A 44, 3782 (1991).

[10] D. Ovsienko, G. Alfintsev, and V. Maslov, J. Cryst. Growth 26, 233 (1974).

[11] S. Huang and M. Glicksmann, Acta Metall. 29, 701 (1981).

[12] J. Bilgram, M. Firmann, and E. Huerlimann, J. Cryst. Growth 96, 175 (1989).

[13] J. Franck and J. Jung, Physica D 23, 259 (1986).

[14] J. Géminard, P. Oswald, D. Temkin, and J. Malthête, Europhys. Lett. 22, 69 (1993).

[15] P. Oswald, J. Malthéte, and P. Pelcé, J. Phys. 50, 2121 (1989).
[16] P. Oswald, J. Phys. (Paris) 49, 2119 (1988).

[17] P. Oswald, J. Phys. (Paris) 49, 1083 (1988).

[18] J. Bechhoefer, P. Oswald, A. Libhaber, and C. Germain, Phys. Rev. A 37, 1691 (1988).

[19] S. Arora, Á. Buka, P. Pallfy-Muhoray, Z. Racz, and R. Vora, Europhys. Lett. 7, 43 (1988).

[20] Á. Buka and N. Éber, Europhys. Lett. 21, 477 (1993).

[21] Á. Buka, T. Tóth Katona, and L. Kramer, Phys. Rev. E 51, 571 (1995).

[22] Y. Carmi, E. Polturak, and S. Lipson, Phys. Rev. Lett. 62, 1364 (1989).

[23] Á. Buka, T. Tóth Katona, and L. Kramer, Phys. Rev. E 49, 5271 (1994).

[24] R. Brownsey and A. Leadbetter, J. Phys. Lett. 42, 135 (1981).

[25] H. Hervet, F. Rondelez, and W. Urbach, in Liquid Crystals, edited by S. Chandrasekhar (Heyden, London, 1980), p. 263.

[26] F. Rondelez, W. Urbach, and H. Hervet, Phys. Rev. Lett. 41, 1058 (1978).

[27] U. Zammit, M. Marinelli, R. Pizzoferrato, F. Scudieri, and S. Martellucci, Phys. Rev. A 41, 1153 (1990).

[28] T. Tóth Katona and Á. Buka, Mol. Cryst. Liq. Cryst. 261, 349 (1995).

[29] S.-L. Wang, R. Sekerka, A. Wheeler, B. Murray, R. Braun, S. Coriell, and G. McFadden, Physica D 69, 189 (1993).

[30] R. Braun, G. McFadden, and S. Coriell, Phys. Rev. E 49, 4336 (1994). 
[31] M. Wortis, in Proceedings of the 1984 Trondheim Summer School, Fundamental Problems in Statistical Mechanics VI, edited by E. Cohen, (North-Holland, Amsterdam, 1985), p. 87.

[32] H. van Beijeren and I. Nolden, in Structure and Dynamics of Surfaces II, edited by W. Schommers and P. van Blankenhagen (Springer-Verlag, Berlin, 1987), p. 259.
[33] We introduced the average growth velocity because in the given sample, different $v$ of the tip could be detected for different germs at the same $\Delta T$. This effect was especially pronounced for $\mathrm{CCH} 4$, where for certain germs the scattering of $v$ around the average value was found to be even $60 \%$.

[34] G. J. Krüger, Phys. Rep. 82, 231 (1982). 